\title{
Searches for Pulsar Planetary Systems
}

\author{
T. Joseph W. Lazio and J. Fischer \\ Naval Research Laboratory, Code 7213, Washington, DC 20375-5351 \\ USA; Joseph.Lazio@nrl.navy.mil; Jacqueline.Fischer@nrl.navy..mil
}

James M. Cordes

Astronomy Dept. and NAIC, Cornell University, Ithaca, NY 14853-6801USA; cordes@astro.cornell.edu

\begin{abstract}
The first extrasolar planetary system was discovered around the pulsar PSR B1257+12, and the planets therein remain the lowest mass exoplanets known (with one whose mass is of order a lunar mass). Pulsar planetary systems will remain the only extrasolar planetary systems within which terrestrial-mass planets can be detected for the near future, and multiple pulsar planetary systems would provide strong circumstantial evidence that terrestrial-mass planets are ubiquitous, and possibly information about the formation of terrestrial-mass planets. We summarize two searches for planetary or proto-planetary systems around pulsars. The first is a series of infrared observations of millisecond pulsars in which the objective is to detect (proto-planetary) disks. The second uses a genetic algorithm - a function optimization method based on evolutionary processes in the natural world-to search for the signatures of planetary perturbations in pulse timing data.
\end{abstract}

\section{Introduction}

The first extrasolar planets discovered were found around the millisecond pulsar PSR B1257+12 (Wolszczan \& Frail 1992). Additional planetary systems around pulsars would provide information about both the late stages of stellar evolution and planetary formation. Moreover, the first two planets discovered in that system are terrestrial-mass planets (with masses of 2.8 and $3.4 \mathrm{M}_{\oplus}$ ) and a lunarmass planet has been discovered subsequently. Thus, pulsar planet systems may also indicate the ubiquity of terrestrial-mass planets.

While a planetary system around a pulsar was unexpected, the various mechanisms proposed for the formation of pulsar planetary systems are similar to those by which planets are thought to form around main sequence stars and rely on the planets forming within an accretion disk around the pulsar (Phinney \& Hansen 1992). Millisecond pulsars themselves are understood to be neutron stars that have been "spun-up" by accretion from a companion (van den Heuvel 1995). Thus, dust disks-representing potential, residual, or failed planetary formation-may exist around millisecond pulsars. 
Despite various efforts, PSR B1257+12 remains the only pulsar known to have planetary companions. Here we describe two attempts to find additional pulsar planetary or proto-planetary systems. The first searches for excess infrared emission characteristic of dust disks while the second makes use of a genetic algorithm (GA) to make a more complete search for orbital perturbations in the received pulses.

\section{Dust Disks around Pulsars}

We observed seven nearby millisecond pulsars at 60 or $90 \mu \mathrm{m}$ with the ISOPHOT instrument (Lemke et al. 1996) on the ISO satellite (Kessler et al. 1996). Table 1 lists these pulsars, their distances, and spin-down luminosities. A rasterpointing observing mode was used around the nominal pulsar position, so that an individual sky position was observed multiple times. Typical rms noise level are approximately $50 \mathrm{mJy}$, though higher values are seen for pulsars close to the Galactic plane. In no case have we identified a source at the location of a pulsar.

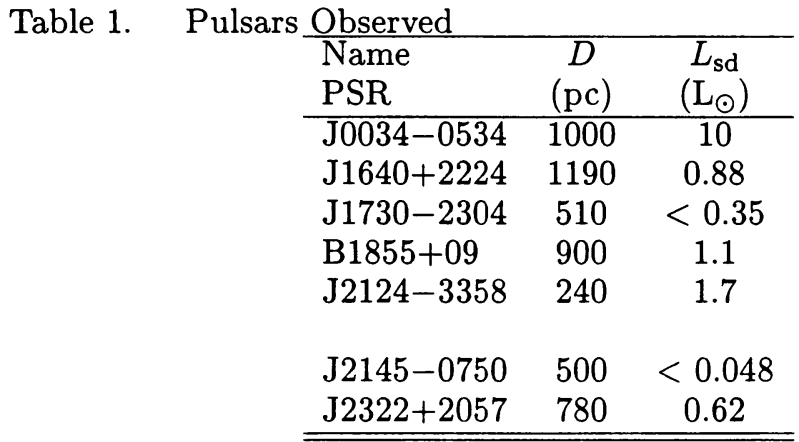

Foster \& Fischer (1996) developed a model for the infrared emission from a circumpulsar dust disk. Their model assumes that a disk of mass $m_{d}$, consisting of particles of a uniform radius $a$, is heated by a fraction $f_{\text {sd }}$ of the pulsar's spindown luminosity $\mathrm{L}_{\mathrm{sd}}$. The particle size is also assumed to be the wavelength at which the blackbody radiation curve of the disk peaks, thereby making $a$ and $m_{d}$ coupled. While simplistic, we believe that this model is acceptable given the uncertainties of the heating mechanism and the environs of a millisecond pulsar.

For $f_{\text {sd }} \sim 1 \%$, dust temperatures are predicted to be $T \approx 10-50 \mathrm{~K}$ for disks having $m_{d} \sim 100 \mathrm{M}_{\oplus}$ and $a \sim 1 \mu \mathrm{m}$ and illuminated by a pulsar with $\mathrm{L}_{\mathrm{sd}} \sim 1 \mathrm{~L}_{\odot}$. These temperatures are lower than those assumed $(\approx 150 \mathrm{~K})$ by Phillips \& Chandler (1994), who estimated disk temperatures by scaling from disks around $\mathrm{T}$ Tauri stars. The lower temperatures result from our assumption of a weaker coupling between the pulsar's spin-down luminosity and the disk, which results in larger disk masses.

In addition to these ISO observations, other infrared and sub-millimeter observations of millisecond pulsars have been conducted $(10 \mu \mathrm{m}$, Foster \& Fischer 1996;850 $\mu \mathrm{m}$, Greaves \& Holland 2000). Unfortunately, there is little overlap in the three samples; most pulsars have been observed at only one wavelength. 
We therefore adopt an approach in which we infer limits on $a$ and $m_{d}$ in the Foster \& Fischer (1996) model for fiducial values of $f_{\text {sd }}$. Assuming $f_{\text {sd }} \sim 1 \%$, we find that particle sizes $a \gtrsim 1 \mu \mathrm{m}$ impose a limit on disk masses $m_{d} \lesssim 3 \mathrm{M}_{\oplus}$ while $a<0.1 \mu \mathrm{m}$ allows $m_{d} \gtrsim 100 \mathrm{M}_{\oplus}$. Obviously, a lower (higher) value of $f_{\text {sd }}$ would produce larger (smaller) allowed regions in the $m_{d^{-}} a$ plane. Measurements at additional wavelengths are required in order to use existing dust disk emission models to place meaningful constraints on the presence circumpulsar dust disks.

\section{A Genetic Algorithm Search}

Available data sets for finding pulsar planetary systems consist of irregularly sampled time series ranging from 5 to $20 \mathrm{yr}$. In addition to any planetary orbital perturbations, the measured pulse times of arrival (TOAs) contain effects from the pulsar spindown, interstellar propagation effects, intrinsic spin noise, and random measurement noise.

In the available data, these other effects produce TOA perturbations that can be modeled as polynomials in time, the coefficients of which can be found using standard linear methods. In contrast orbital motion perturbations involve a non-linear function of the orbital period, $T$, and three orbital elements describing the shape of the orbit-phase, $\phi$, eccentricity, $e$, and argument of periastron, $\omega$; the semi-major axis and inclination combine to give the size of the (pulsar's) orbit or the amplitude of the TOA perturbation, which enters as a linear coefficient. ${ }^{1}$ For a single planet in a circular orbit, $\phi$ and $\omega$ are degenerate, $e=0$, and the orbital TOA perturbation depends upon 3 parameters, 2 nonlinearly. Solving such a problem is relatively easy. However, fitting for

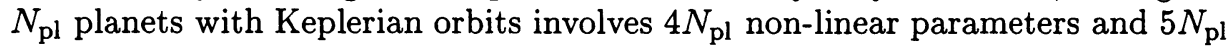
total. The resulting parameter space may be quite complicated with the global minimum surrounded by many local minima.

We desire a search of parameter space that is efficient yet complete-a relatively small number of function evaluations yet finding the global minimum. The essential idea of the GA is to search a complicated parameter space making use of an optimization mechanism from the natural world: Successive applications of crossover (sexual reproduction), mutation, and survival of the fittest operators to an initial set of guesses for the model parameters.

To this end, we use a "genetic code" for a model in which its non-linear parameters, $\{T, \phi, e, \omega\}$, are represented as binary numbers; given an estimate of these parameters, the remaining (linear) model parameters can be found using standard linear methods. The set of binary numbers encoding one particular set of model parameters forms an "organism."

Our GA begins with a fixed number of organisms (a "population") distributed randomly throughout parameter space. The "fitness" for each organism (i.e., the agreement between the model encoded in the binary parameters and the data) is evaluated. The GA then produces a series of new "generations"

\footnotetext{
${ }^{1} \mathrm{~A}$ sixth orbital element describes the orbit's orientation about the line of sight, an angle to which TOA measurements are insensitive.
} 
of this original population. In each generation, the fitness for each organism is evaluated after application of the following operations:

Survival of the Fittest In natural selection, the most fit organisms are those most likely to survive and reproduce. To reproduce this effect, we select fit organisms preferentially; we do not select the most fit organisms exclusively to allow for the possibility that a minor subsequent change in an organism could produce a considerable improvement in its agreement with the data.

Crossover Sexual reproduction in biological organisms introduces (minor) variations into a gene pool. In the same fashion, the GA uses crossover to explore the local neighborhood of parameter space in which good models have been found. A subset of the successful organisms (i.e., those that have survived) are chosen to interchange portions of their encoded models.

Mutation Mutations introduce (usually major) variations into a gene pool. Likewise, the GA uses mutations to search distant regions of parameter space and thereby avoid becoming stuck in local minima. A subset of the successful organisms (after crossover) are chosen for a single random bit flips (i.e., 0 is changed to 1 or vice versa in the binary encoding). Like the biological case, mutated organisms are usually, but not always, far less fit.

We applied our GA to a pulsar timing database consisting of 21 objects. Time series analyzed contained 200-500 samples over 7-12 yr. The only pulsars for which planets might make substantial contributions to the TOA time series are PSR B1911-04 and PSR B1929+10, which may be orbited by Earth-mass planets with orbital periods of 7-12 yr. Upper limits on the mass and orbital period of planetary companions to the other pulsars are $m \sin i \approx 0.1 \mathrm{M}_{\oplus}$ to $10 \mathrm{M}_{\oplus}$. However, unambiguous discrimination of planetary perturbations from intrinsic spin noise is difficult. Longer, more densely sampled TOA time series are needed in all cases to confirm these possible planetary detections for PSR B1911-04 and PSR B1929+10 and improve the limits for other pulsars.

Acknowledgments. We thank D. Nice for providing us with some of the pulsar time series and Z. Arzoumanian and B. Gladman for many helpful discussions. J. Novak, T. Metcalfe, M. Donegan, P. Piper, and L. Dzombar assisted with various aspects of the GA project. We thank the organizers of the ISOPHOT Workshop on PHT32 Oversampled Mapping, particularly R. Tuffs, C. Gabriel, N. Lu, and B. Schulz, and R. Tuffs for his software. The results reported here are based on observations with ISO, an ESA project with instruments funded by ESA Member States and with the participation of ISAS and NASA. The ISOPHOT data were reduced using PIA, developed by the ESA Astrophysics Division and the ISOPHOT consortium with the collaboration of the Infrared Analysis and Processing Center (IPAC) and the Instituto de Astrofísica de Canarias. This work was supported by the NASA ISO grant program and by NSF grant AST 9819931. The NAIC is operated by Cornell University under a cooperative agreement with the NSF. A portion of this work was performed while TJWL held a National Research Council Associateship at the NRL. Basic research in radio astronomy at the NRL is supported by the Office of Naval Research. 


\section{References}

Foster, R. S. \& Fischer, J. 1996, ApJ, 460, 902

Greaves, J. S. \& Holland, W. S. 2000, MNRAS, 316, L21

Kessler, M. F., et al. 1996, A\&A, 315, L27

Lemke, D., et al. 1996, A\&A, 315, L64

Phillips, J. A. \& Chandler, C. J. 1994, ApJ, 420, L83

Phinney, E. S. \& Hansen, B. M. S. 1992, in Planets around Pulsars, eds. J. A. Phillips, S. E. Thorsett, \& S. R. Kulkarni (San Francisco: ASP) p. 371

van den Heuvel, E. P. J. 1995, J. Astrophys. Astron., 16, 255

Wolszczan, A. \& Frail, D. A. 1992, Nature, 355, 145 


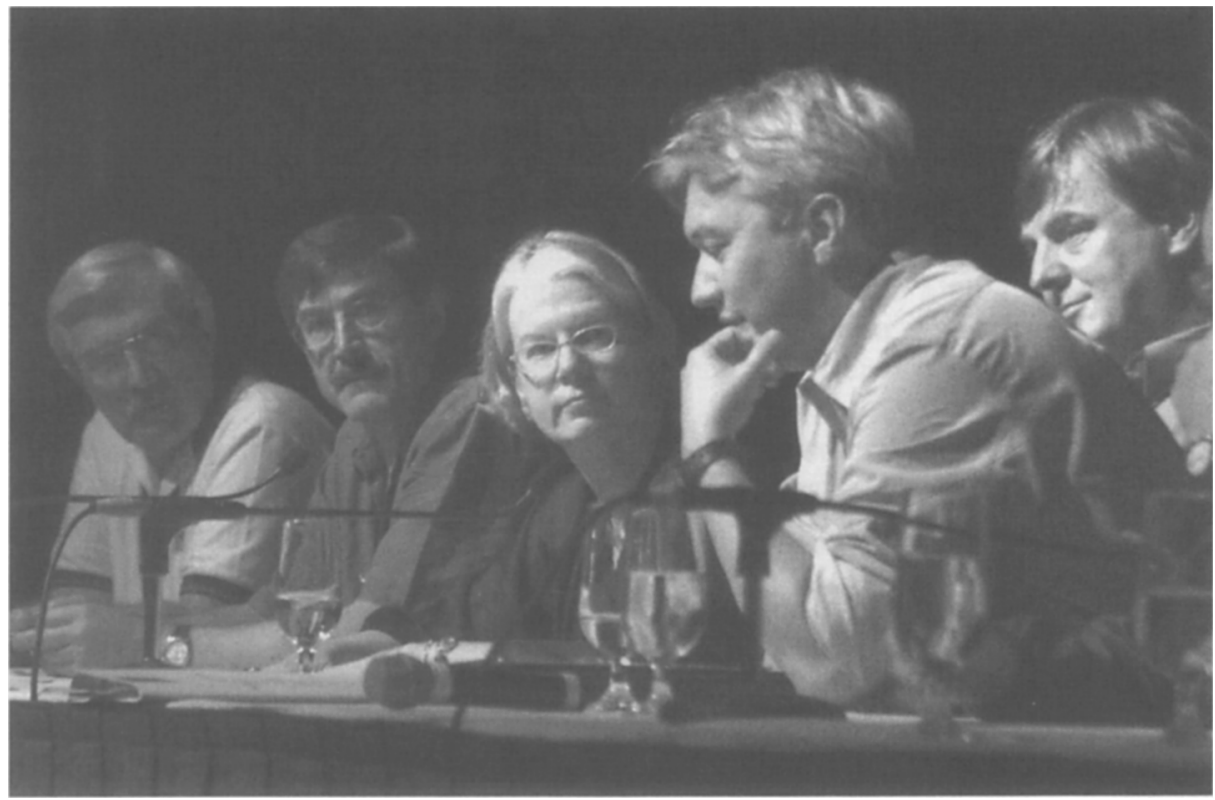

Panel discussion: Everett Gibson, Paul Davies, Jill Tarter, Chris Chyba, \& Ray Norris (photo: Seth Shostak) 